\title{
The 21 Centimeter Forest: Radio Absorption Spectra as Probes of Minihalos before Reionization
}

\section{Citation}

Furlanetto, Steven R., and Abraham Loeb. 2002. “The 21 Centimeter Forest: Radio Absorption Spectra as Probes of Minihalos before Reionization." The Astrophysical Journal 579 (1): 1-9. https://doi.org/10.1086/342757.

\section{Permanent link}

http://nrs.harvard.edu/urn-3:HUL.InstRepos:41393329

\section{Terms of Use}

This article was downloaded from Harvard University's DASH repository, and is made available under the terms and conditions applicable to Other Posted Material, as set forth at http:// nrs.harvard.edu/urn-3:HUL.InstRepos:dash.current.terms-of-use\#LAA

\section{Share Your Story}

The Harvard community has made this article openly available.

Please share how this access benefits you. Submit a story.

Accessibility 


\title{
The $21 \mathrm{~cm}$ Forest: Radio Absorption Spectra as a Probe of the Intergalactic Medium Before Reionization
}

\author{
Steven R. Furlanetto \& Abraham Loeb \\ Harvard-Smithsonian Center for Astrophysics, 60 Garden St., Cambridge, MA 02138; \\ sfurlanetto@cfa.harvard.edu, aloeb@cfa.harvard.edu
}

\begin{abstract}
We study the absorption along lines of sight toward high- $z$ radio sources caused by the $21 \mathrm{~cm}$ transition of neutral hydrogen in the intergalactic medium (IGM) before reionization. Using semi-analytic methods, we compute the number density of observable features caused by both "minihalos" (bound objects that are unable to cool efficiently because of their small virial temperatures) and protogalactic disks. We show that both sets of features should be observable by the next generation of low-frequency radio telescopes, including the Low Frequency Array and the Square Kilometer Array, provided that sufficiently bright background sources exist. The statistics of minihalo absorption features seen along lines of sight to radio-loud quasars offer a way to measure the evolution of the radiation background and the IGM temperature with cosmic time. Intersections with disks are much less common, but also cause much deeper absorption features that would be visible in the spectra of both radio-loud quasars and gamma-ray bursts (GRBs). The absorption feature caused by HI in the host galaxy of a GRB should be observable, offering a route to spectroscopically determine the burst redshift.
\end{abstract}

Subject headings: cosmology: intergalactic medium, structure formation - galaxies: active, radio, radio lines

\section{Introduction}

Despite its apparent simplicity, effective observational probes of the intergalactic medium (IGM) at high redshifts are difficult to find. Between recombination at $z \sim 1000$ and reionization at $z \sim 6$, the IGM was almost entirely neutral. In such a medium, the optical depth to $\operatorname{Ly} \alpha$ absorption at the frequency corresponding to a redshift $z$ is $\tau_{\alpha} \approx$ $6.45 \times 10^{5} x_{\mathrm{HI}}[(1+z) / 10]^{3 / 2}$ (Gunn \& Peterson 1965), where $x_{\mathrm{HI}}$ is the neutral fraction and 
where we have assumed the currently favored cosmological parameters (see below). This enormous optical depth renders the Ly $\alpha$ forest, which is the most important tool for studying the (highly ionized) IGM at low and moderate redshifts, almost useless for detailed studies of the pre-reionization IGM. For example, the detection of a complete absorption trough shortward of the Ly $\alpha$ resonance wavelength in the spectrum of the quasar SDSS 1030+0524 may be the first evidence for the end of the reionization era (Becker et al. 2001). However, studies of rest-frame UV and optical quasar spectra are unable to place stronger constraints on the neutral fraction than $x_{\mathrm{HI}} \gtrsim 10^{-2}$ (Fan et al. 2002). Attempts to constrain other parameters, such as the thermal state of the IGM, the radiation background, or the density structure, are also compromised by the very large optical depth at the Ly $\alpha$ resonance.

Clearly, studies of the Ly $\alpha$ forest are not sufficient for an in-depth understanding of the IGM before and during reionization. An alternative is to observe a weaker transition at the other extreme of optical depth: the $21 \mathrm{~cm}$ hyperfine transition of neutral hydrogen. To date, there have been several theoretical studies of emission or absorption of the neutral IGM against the cosmic microwave background (CMB) in this transition (Scott \& Rees 1990; Kumar et al. 1995; Madau et al. 1997; Tozzi et al. 2000; Shaver et al. 1999; Iliev et al. 2002). However, the predicted signals are extremely weak. Even the next generation of radio telescopes, such as the Square Kilometer Array ${ }^{1}$ (SKA), will only be able to detect exceptionally large objects (e.g., Scott \& Rees 1990; Kumar et al. 1995). Another strategy is to study the angular structure of the emission in order to constrain statistically the structure formation process at high redshift (e.g., Tozzi et al. 2000; Iliev et al. 2002). However, source confusion with low- $z$ faint radio sources (Di Matteo et al. 2002) and free-free emission by the intergalactic medium (Loeb 1996) are likely to compromise such attempts.

Alternatively, Carilli et al. (2002) have pointed out that luminous high-redshift radioloud quasars serve as ideal background sources against which absorption by intervening gas can be seen: with a bright background source it becomes much easier to identify the weak absorption features expected to be produced by the IGM. Along such lines of sight one can map the "21 cm forest" of redshifted hyperfine absorption lines and hence study the neutral IGM over the redshift range $6 \lesssim z \lesssim 10$. Using a simulation, Carilli et al. (2002) found that the high- $z$ analog of the Ly $\alpha$ forest of filaments and other overdense regions produces recognizable absorption features in the spectrum of a radio-loud quasar. They also demonstrated that such observations are feasible with an instrument similar to current designs for the SKA, provided that sufficiently bright radio-loud quasars exist at these early

\footnotetext{
${ }^{1}$ See, e.g., http://www.usska.org/main.html.
} 
times.

Large scale numerical simulations, such as those studied by Carilli et al. (2002), are unable to resolve collapsed gas clouds on the smallest mass scales, although these clouds represent the most abundant clumps in the high-redshift IGM. In this paper we complement their study by exploring semi-analytically the statistics of these compact absorbing systems. Our semi-analytic approach allows us to examine the sensitivity of the results to changes in the input parameters and to identify the physical quantities that are best measured by radio absorption spectra. As we demonstrate in the following sections, $21 \mathrm{~cm}$ absorption spectra probe the radiation background and thermal state of the IGM at high redshifts and therefore could be instrumental in testing models of structure formation and reionization.

The smallest bound objects in the IGM are commonly termed "minihalos;" their virial temperatures are below the threshold for atomic hydrogen line cooling and so (in the absence of a substantial abundance of molecular hydrogen, $\mathrm{H}_{2}$ ) they cannot cool and collapse to form protogalaxies. Although minihalos cannot form stars, they are important for determining the mean clumping factor of the IGM and for screening ionizing radiation from other objects (Barkana \& Loeb 2002). Their properties are sensitive to the uncertain presence of molecular hydrogen; if $\mathrm{H}_{2}$ forms in sufficient quantities it can act as a cooling channel for these minihalos (Barkana \& Loeb 2001, and references therein), strongly suppressing their number density (and hence increasing the global star formation rate). Detection of these halos would provide important insights into the physics of hierarchical structure formation prior to reionization.

Halos with virial temperatures above the hydrogen line cooling threshold will collapse to form stars. Thus we expect some fraction of all lines of sight to penetrate protogalactic disks. Such absorption systems would be the pre-reionization analogs of damped Ly $\alpha$ absorbers. While disk intersections are rare, they can provide important information about the state of the neutral hydrogen in high- $z$ galaxies, including the distribution of disk masses, star formation rates, and gas temperature.

In $\S 2$ we derive the optical depth to redshifted $21 \mathrm{~cm}$ radiation both in the diffuse IGM and in collapsed objects. We show our numerical results in $\S 3$ and conclude in $\S 4$. Throughout the discussion we assume a flat, $\Lambda$-dominated cosmology, with density parameters $\Omega_{m}=0.3, \Omega_{\Lambda}=0.7, \Omega_{b}=0.05$, in matter, cosmological constant, and baryons, respectively. In the numerical calculations, we assume $h=0.7$, where the Hubble constant is $H_{0}=100 h \mathrm{~km} \mathrm{~s}^{-1} \mathrm{Mpc}^{-1}$. 


\section{Model}

\subsection{The Diffuse IGM}

The optical depth to redshifted hyperfine absorption by the neutral IGM is (Field 1959a; Madau et al. 1997)

$$
\begin{aligned}
\tau_{\nu} & =\frac{3 c^{3} h_{P} A_{10} n_{H I}(z)}{32 \pi k \nu_{0}^{2} T_{S} H(z)} \\
& \approx 10^{-2}\left[\frac{T_{\mathrm{CMB}}(z)}{T_{S}}\right]\left(\frac{\Omega_{b} h}{0.035}\right)\left[\left(\frac{0.3}{\Omega_{m}}\right)\left(\frac{1+z}{10}\right)\right]^{1 / 2} x_{\mathrm{HI}}
\end{aligned}
$$

Here $h_{P}$ is Planck's constant, $k$ is Boltzmann's constant, $\nu_{0}=1420.4 \mathrm{MHz}=\nu /(1+z)$ is the rest-frame hyperfine transition frequency, $A_{10}=2.85 \times 10^{-15} \mathrm{~s}^{-1}$ is the spontaneous emission coefficient for the transition, $T_{S}$ is the spin temperature of the IGM, $T_{\mathrm{CMB}}=$ $2.73(1+z) \mathrm{K}$ is the CMB temperature at redshift $z$, and $n_{H I}$ is the neutral hydrogen density at this redshift. In the second equality, we have assumed sufficiently high redshifts such that $H(z) \approx H_{0} \Omega_{m}^{1 / 2}(1+z)^{3 / 2}$, which is well-satisfied in the era we study $\left(z_{r} \gtrsim 6\right)$. Because reionization is expected to be sudden (e.g., Gnedin 2000) we take a neutral fraction $x_{\mathrm{HI}}=1$ throughout our discussion.

The HI spin temperature (i.e., the excitation temperature of the hyperfine transition) is (Field 1958)

$$
T_{S}=\frac{T_{\mathrm{CMB}}+y_{\alpha} T_{\alpha}+y_{c} T_{K}}{1+y_{\alpha}+y_{c}} .
$$

Here $T_{K}$ is the HI kinetic temperature and and $T_{\alpha}$ is the Ly $\alpha$ color temperature (see Madau et al. 1997 for a precise definition). In the conditions pertaining to both the IGM and collapsed objects, $T_{\alpha}=T_{K}$ (Field 1959b). The third term describes collisional excitation of $T_{S}$, while the second describes the Wouthuysen-Field effect, in which Ly $\alpha$ pumping couples the spin temperature to the temperature of the radiation field (Wouthuysen 1952; Field 1958). The coupling from collisions is

$$
y_{c}=\frac{C_{10}}{A_{10}} \frac{T_{\star}}{T_{K}}
$$

where $C_{10}$ is the collisional de-excitation rate of the higher-energy triplet hyperfine level and $T_{\star}=h_{P} \nu_{0} / k=0.068 \mathrm{~K}$. We use a fit to the results of Allison \& Dalgarno (1969) for $C_{10}$ at $T_{K}<1000 \mathrm{~K}$, with an extrapolation $C_{10} \propto T_{K}^{-0.33}$ to higher temperatures. The Wouthuysen-Field coupling constant is

$$
y_{\alpha}=\frac{P_{10}}{A_{10}} \frac{T_{\star}}{T_{\alpha}},
$$


where $P_{10}$ is the indirect de-excitation rate of the triplet level due to absorption of a Ly $\alpha$ photon followed by decay to the singlet level. For a diffuse Ly $\alpha$ background, Madau et al. (1997) showed that

$$
P_{10} \approx 1.3 \times 10^{-12} J_{-21} \mathrm{~s}^{-1}
$$

where $J_{-21}$ is the intensity of the background radiation field at the Ly $\alpha$ frequency in units of $10^{-21} \mathrm{erg} \mathrm{cm}^{-2} \mathrm{~s}^{-1} \mathrm{~Hz}^{-1} \mathrm{sr}^{-1}$. Within the diffuse IGM, the gas density is small enough that collisions do not strongly couple $T_{S}$ and $T_{K}$. However, collisions can effectively couple the two quantities within collapsed objects. Ly $\alpha$ pumping couples the two temperatures in a density-independent manner, becoming effective when $J_{-21} \gtrsim 1$. Below we show results for several values of $J_{-21}$, because the UV radiation field before reionization is an unknown quantity, likely varying strongly with both position and time. For reference, the simulation used by Carilli et al. (2002) yields $J_{-21}(z=10) \approx 0.8$ and $J_{-21}(z=8) \approx 4$.

The spin temperature and optical depth therefore depend on the kinetic temperature of the IGM. Once Thomson scattering with the CMB becomes inefficient at the thermal decoupling redshift $z_{d} \sim 140$, the IGM cools adiabatically as the universe expands (Barkana \& Loeb 2001) until the first objects collapse. As soon as this occurs, radiative (and mechanical) feedback from these objects quickly heats the IGM. Because the radiation background prior to reionization is still unconstrained by observations, estimates of the temperature rely on simulations. We choose a particular form for the temperature evolution that roughly matches the mass-weighted temperature found in the simulation of Carilli et al. (2002):

$$
T_{\text {IGM }}(z)=10^{-0.25 z+5.5} \mathrm{~K} .
$$

This simple form has two convenient properties. First, heating begins at $z \sim 18$, close to the time when the first stars are expected to form. Second, it yields $T_{\mathrm{IGM}}(z=6)=10^{4} \mathrm{~K}$, close to the temperature of a photo-ionized medium at approximately the time when reionization may have occurred. We neglect any spatial variation in the temperature field, as is likely to occur in filaments and other overdense regions. For contrast, we include below some results for zero heating and for a temperature that is smaller than that given in equation (6) by a factor of 10 .

\subsection{Minihalos}

In cold dark matter models, structure forms hierarchically (i.e., low-mass halos collapse first and the characteristic mass scale increases with cosmic time). The smallest halos cannot, however, form stars because they cannot cool efficiently: without metals, atomic hydrogen line cooling is only effective for halos with a virial temperature $T_{\text {vir }} \gtrsim 10^{4} \mathrm{~K}$. Here, $T_{\text {vir }}$ is 
defined as in equation (26) of Barkana \& Loeb (2001). We use the term minihalo to refer to collapsed objects with $T_{\text {vir }}<10^{4} \mathrm{~K}$. This condition sets the upper mass limit for minihalos; the lower mass limit is determined by the Jeans mass or, more precisely, the time-averaged Jeans mass $M_{\text {fil }}$ (Gnedin \& Hui 1998) using the IGM temperature given in equation (6). If molecular hydrogen is present, cooling becomes more effective (Ricotti et al. 2002), but most models predict that even a small UV background will efficiently dissociate $\mathrm{H}_{2}$ in these halos (Haiman et al. 1997; Ciardi et al. 2000; Haiman et al. 2000).

We compute the optical depth $\tau_{\nu}$ of the $21 \mathrm{~cm}$ transition along a line of sight through a minihalo with impact parameter $\alpha$. For the remainder of this section, $\nu$ refers to the frequency of the background radiation at the position of the minihalo (i.e., not including the cosmological redshift between the observer and the minihalo). Each collapsed object is surrounded by an accretion shock. Inside the shock, we assume that the gas overdensity equals that of the dark matter, which we take to have the universal NFW profile of Navarro, Frenk, \& White (1997). In reality, gas pressure will flatten the core of the gas profile relative to the dark matter, but we find that lines of sight passing through the affected regions are so rare that they make only a negligible difference to our final results.

Outside of the virialization shock, each halo is surrounded by a region of infalling gas. In an Einstein-de Sitter universe, the structure of the infall region can be described by a selfsimilar solution (Bertschinger 1985). At the redshifts we consider $(6 \lesssim z \lesssim 10)$, deviations from an Einstein-de Sitter universe are small, and the solution is approximately valid. The Bertschinger (1985) profile fixes the shock to be at a constant overdensity relative to the background medium: the shock radius $r_{\mathrm{sh}} \approx 4 r_{\text {vir }} / 3$, with a slight dependence on cosmology and redshift. Here $r_{\text {vir }}$ is the virial radius, as defined in equation (24) of Barkana \& Loeb (2001). Similarly, the turnaround radius (i.e., the radius at which the gas is at rest with respect to halo) is $r_{t a} \approx 3.85 r_{v i r}$. We scale the velocity field to match the Hubble flow and the gas density to match the mean IGM density at large radii. Unfortunately, the Bertschinger (1985) profile has a much steeper central cusp $\left(\rho \propto r^{-2.25}\right)$ than the NFW profile. We therefore continue to use an NFW profile within the accretion shock in order to avoid seriously overcounting the number of high-optical depth lines of sight. As a result, the density jump condition at the shock is only satisfied to $\sim 10 \%$ for the halos we study. However, because all of the quantities of interest are integrated along the line of sight, this error makes little difference to our results.

The HI atoms are assumed to have a Maxwellian distribution at each point. For $r \leq r_{\text {sh }}$ we set $T_{K}=T_{\text {vir }}$, while for $r>r_{\mathrm{sh}}$, we set $T_{K}=T_{\mathrm{IGM}}$. Note that we therefore ignore temperature variations within the shocked region. The Bertschinger (1985) solution gives a postshock temperature which is a factor of $\sim 3$ smaller than $T_{\text {vir }}$ and thus slightly increases 
the optical depths. We also ignore the heating of the gas as it is compressed in the infall region; in the adiabatic limit, this leads to at most a factor $\sim 2.5$ error in the temperature. However, because the overdensity in the infall region is not large, the effects on the total optical depths are small. The spin temperature is then determined locally via equation (2).

Once the halo profile is specified, the optical depth at a frequency $\nu$ along a line of sight with impact parameter $\alpha$ from the halo center is

$$
\tau_{\nu}=\frac{3 h_{P} c^{3} A_{10}}{32 \pi k \nu_{0}^{2}} \int_{-\infty}^{\infty} d R \frac{n_{H I}(r)}{T_{s}(r) \sqrt{\pi} b(r)} \exp \left\{-\frac{\left[v(\nu)-v_{\mathrm{LOS}}(\alpha, R)\right]^{2}}{b^{2}(r)}\right\} .
$$

Here, $r^{2}=\alpha^{2}+R^{2}, v(\nu)=c\left(\nu-\nu_{0}\right) / \nu_{0}, v_{\mathrm{LOS}}(\alpha, R)$ is the infall velocity projected along the line of sight, and $b^{2}(r)=2 k T_{K}(r) / m_{p}$ is the Doppler parameter of the gas. For $\nu$ sufficiently far from $\nu_{0}$, the exponential factor is non-zero only in the diffuse IGM where $n_{H I}, T_{K}$, and $T_{S}$ are constants. For these frequencies, the integral becomes $n_{H I} /\left[T_{S} H(z)\right]$ due to the Hubble flow, and we recover equation (1).

Sample optical depth profiles for a $M_{h}=5 \times 10^{6} \mathrm{M}_{\odot}$ minihalo at $z_{h}=10$ are shown in Figure 1. The solid curves assume a heated IGM and $J_{-21}=0$ while the dotted lines show analogous results for $J_{-21}=10$. Each set of profiles assumes $\alpha=0.3,1.3$, and $3 r_{\text {vir }}$, from top to bottom. In all cases, the optical depth at large $\nu-\nu_{0}$ is simply that of the IGM, which is strongly suppressed for a strong $T_{S}-T_{K}$ coupling through a Ly $\alpha$ background. Careful examination of the $\alpha=3 r_{\text {vir }}$ curves reveals that $\tau_{\nu}<\tau_{\text {IGM }}$ for moderately large $\nu-\nu_{0}$. This occurs because the velocity gradients within the infall region exceed that of the Hubble flow, and so for a range of frequencies the absorbing column is actually smaller than that of the diffuse IGM.

As is evident from Figure 1, the line width varies with impact parameter. For $\alpha \lesssim r_{\text {vir }}$, the dominant contribution to $\tau_{\nu}$ comes from shocked gas within the halo, and so the line profile is close to a Gaussian with a width determined by $T_{\text {vir }}$. For $\alpha \gg r_{\text {vir }}$, the line of sight does not pass through shocked gas and so the profile is approximately a Gaussian of width $T_{\text {IGM }}$. For $\alpha \gtrsim r_{\text {vir }}$, the line of sight passes close to the shock, and the profile is best fit by an effective temperature $T_{\text {eff }}=0.5\left(T_{\text {IGM }}+T_{\text {vir }} / 3\right)$. [The factor of $1 / 3$ results from the infall velocities at the shock in the Bertschinger (1985) profile; see the paragraph above equation (7).] The observed line width is then

$$
\Delta \nu_{\mathrm{obs}}=3.8\left(\frac{T_{\mathrm{eff}}}{10^{3} \mathrm{~K}}\right)^{1 / 2}\left(\frac{1+z_{h}}{10}\right)^{-1} \mathrm{kHz}
$$

where $T_{\text {eff }}$ is chosen as described above and $z_{h}$ is the redshift of the minihalo.

Another quantity of interest is a measure of the total absorption due to the halo, or the 
"equivalent width." We define the intrinsic equivalent width through the relation

$$
\frac{\langle\Delta \nu\rangle_{\mathrm{int}}}{2}=\int_{\nu_{0}}^{\infty}\left(1-e^{-\tau_{\nu}}\right) d \nu-\int_{\nu_{0}}^{\infty}\left(1-e^{-\tau_{\mathrm{IGM}}}\right) d \nu,
$$

where $\tau_{\text {IGM }}$ is given by equation (1). This measures the excess amount of absorbed flux due to the minihalo on top of the absorption due to the diffuse IGM. Because of the cosmological redshift, the observed equivalent width $\langle\Delta \nu\rangle_{\mathrm{obs}}=\langle\Delta \nu\rangle_{\mathrm{int}} /\left(1+z_{h}\right)$.

The observed equivalent width as a function of impact parameter is shown for several $z_{h}=8$ halos in Figure 2. Solid curves show results for $M_{h}=10^{7}, 10^{6}$, and $10^{5} \mathrm{M}_{\odot}$ from top to bottom, with $J_{-21}=0$ and a heated IGM. The dotted line shows results for $M_{h}=10^{6} \mathrm{M}_{\odot}$ and no IGM heating, while the dashed line shows results for the same mass, full IGM heating, and a radiation background $J_{-21}=10$. The steep drop at $\alpha / r_{\text {vir }} \approx 1.3$ occurs because of the density drop past the accretion shock. Note that the IGM temperature has little effect on the equivalent width. Smaller halos have larger central equivalent widths (because such halos are cooler, and $\tau_{\nu} \propto T_{S}^{-1}$ ) but smaller equivalent widths at the most probable impact parameters because their column density is smaller. A cold IGM slightly increases the equivalent width at large $\alpha$ because more of the absorbing gas is located within the infall region of the halo, where the density is above the mean IGM density. On the other hand, the radiation background has a pronounced effect on the equivalent widths, particularly at large $\alpha$. By effectively coupling $T_{S}$ to $T_{\text {vir }}$ or $T_{\text {IGM }}$ in the outskirts of the halo where collisions are ineffective, such a radiation field drastically decreases the optical depth contributed by these regions.

\subsection{Galactic Disks}

Halos with $T_{\text {vir }}>10^{4} \mathrm{~K}$ can cool through atomic line radiation and are expected to collapse and form protogalactic objects. We assume here that these objects form disks. We adopt the exponential disk structure of Mo et al. (1998), in which

$$
\begin{array}{r}
\Sigma(\alpha)=\frac{M_{d}}{2 \pi R_{d}^{2}} e^{-\alpha / R_{d}}, \\
R_{d}=\frac{1}{\sqrt{2}} j_{d} \lambda r_{\mathrm{vir}} .
\end{array}
$$

Here $\Sigma$ is the surface density of the disk, $R_{d}$ is the disk scale length, $M_{d}$ is the disk mass,

$j_{d}$ is the specific angular momentum of the baryons in units of their initial value upon halo collapse, and $\lambda$ is the spin parameter. Mo et al. (1998) found that $j_{d}=1$ was required in order to match observational data (implying no substantial loss of baryonic angular momentum 
to the dark matter), given the fact that N-body simulations show that $\lambda$ has a lognormal distribution with a mean $\bar{\lambda}=0.05$. We will assume for simplicity that all disks have this mean spin parameter, $\bar{\lambda}$.

The primary contribution to HI absorption in galaxies comes from the cold neutral medium. We assume that a fraction $f_{d}$ of the total baryonic mass of the halo, $\left(\Omega_{b} / \Omega_{m}\right) M_{h}$, is in this phase. (The remainder can be in stars, the hot interstellar medium, or a hot component of the halo.) We assume for simplicity that the cold gas is smoothly distributed throughout the disk (although in reality it is likely to be bound up in small, dense clouds dispersed throughout the disk). We take a spin temperature $T_{S}=10^{3} \mathrm{~K}$, as inferred from observations of damped Ly $\alpha$ absorbers at lower redshifts (Carilli et al. 1996). We further assume that the spin and kinetic temperatures are coupled, as expected for the radiation background in a typical galaxy. The optical depth is then

$$
\tau_{\nu}=\tau_{\mathrm{IGM}}+\tau_{0} \exp \left[-\frac{v^{2}(\nu)}{b^{2}\left(T_{K}\right)}\right],
$$

where $\tau_{0}=\tau_{0}^{c} e^{-\alpha / R_{d}}$ and

$$
\begin{aligned}
\tau_{0}^{c}= & 15.1 h^{4 / 3}\left(\frac{f_{d}}{0.5}\right)\left(\frac{\Omega_{b}}{0.05}\right)\left(\frac{\Omega_{m}}{0.3}\right)^{-1 / 3}\left(\frac{T_{S}}{10^{3} \mathrm{~K}}\right)^{-3 / 2} \\
& \times\left(\frac{\bar{\lambda}}{0.05}\right)^{-2}\left(\frac{M_{h}}{10^{8} \mathrm{M}_{\odot}}\right)^{1 / 3}\left(\frac{1+z}{10}\right)^{2} .
\end{aligned}
$$

In this expression, we have assumed a random distribution of disk inclinations and taken the average path length through an inclined disk (so that $\Sigma_{0} \rightarrow \pi^{2} / 4 \Sigma_{0}$ ). We have ignored the modifications to $\tau_{\text {IGM }}$ caused by the velocity gradient and overdensity of the infall region, which differs slightly from the Hubble flow (and therefore, at a given velocity offset, the column of neutral hydrogen differs from that of the unperturbed IGM). Typically, the large optical depths of lines of sight through galaxies make such subtle differences unimportant. However, note that disks are likely to be in complicated environments, both because of feedback from the collapsed object and because high-redshift protogalaxies are expected to be highly clustered. Feedback can heat and ionize the region around the disk, reducing the IGM absorption well below the nominal $\tau_{\text {IGM }}$. The equivalent width can be calculated from equation (9); with our assumption of a constant $T_{S}=T_{K}$ within each system, it can be written analytically as

$$
\langle\Delta \nu\rangle_{\text {int }}=34 \mathrm{kHz}\left(\frac{T_{S}}{10^{3} \mathrm{~K}}\right)^{1 / 2} \sum_{n=1}^{\infty} \frac{(-1)^{n-1} \tau_{0}^{n}}{n ! \sqrt{n}} .
$$




\section{Results}

\subsection{Number Densities}

Using our model we can compute the density of lines of a given peak optical depth or equivalent width in a spectrum. For a quantity $\zeta$ (either $\tau_{0}$ or equivalent width), the number of systems intersected with $\zeta>\zeta_{0}$ per redshift interval is

$$
\frac{d N\left(>\zeta_{0}\right)}{d z}=(1+z)^{2} \frac{d r}{d z} \int_{M_{\min }}^{M_{\max }} d M_{h} \frac{d n_{h}}{d M_{h}} A\left(M_{h}, z, \zeta_{0}\right) .
$$

Here $d r / d z$ is the comoving length element per unit redshift, $d n_{h} / d M_{h}$ is the halo mass function (or number of halos at redshift $z$ with mass between $M_{h}$ and $M_{h}+d M_{h}$ per comoving volume), and $A\left(M_{h}, z, \zeta_{0}\right)$ is the cross-sectional area of a halo (in physical coordinates) with the specified mass and redshift subtended by lines of sight for which $\zeta>\zeta_{0}$. For minihalos, $M_{\text {min }}=M_{\text {fil }}$ and $M_{\max }$ is determined by the cooling criterion. For disks, $M_{\min }$ is determined by cooling and $M_{\max }=\infty$. In both cases, we use $d n_{h} / d M_{h}$ given by the Press-Schechter formalism (Press \& Schechter 1974), with the modifications suggested by Jenkins et al. (2001) in order to better match the mass function found by numerical simulations. For disk calculations, we again assume a random distribution of disk inclinations; the effective area of each disk is therefore $4 / \pi^{2}$ times the area of the disk viewed face-on. In this case the area is simply

$$
A\left(M_{h}, z, \tau_{0}\right)=\frac{2 \bar{\lambda}^{2} r_{\mathrm{vir}}^{2}}{\pi} \ln ^{2}\left(\frac{\tau_{0}^{c}\left(M_{h}, z\right)}{\tau_{0}}\right)
$$

Figure 3 shows $d N\left(>\tau_{0}\right) / d z$, the number of objects intersected per redshift interval with a central optical depth greater than $\tau_{0}$. The dashed, solid, and dotted curves show results for $z=10,8$, and 6 , respectively. All minihalo calculations assume a heated IGM and $J_{-21}=1$. The density of minihalo lines decreases with cosmic time because the minimum halo mass increases as $T_{\mathrm{IGM}}$ increases. Note that the evolution with redshift is particularly pronounced for large $\tau_{0}$.

It is clear that minihalo absorption systems are much more common than disks at low optical depths, but the rare high-optical depth features are dominated by collapsed disks. The flat disk distribution results from the exponential dependence of the optical depth on impact parameter. The primary uncertainty in our disk calculation is $T_{S}$. A single cold cloud in the Milky Way typically has $T_{S} \sim 100 \mathrm{~K}$ (Dickey \& Lockman 1990), but observations of moderate-redshift absorbers suggest $T_{S} \sim 10^{3} \mathrm{~K}$ (Carilli et al. 1996). If $T_{S}$ is smaller than we assume, the optical depth through the disk increases substantially $\left[\tau_{0} \propto T_{S}^{-3 / 2}\right.$; see equation (13)]. However, as equation (16) shows, the distribution varies only logarithmically with 
$\tau_{0}^{c}$, and so the corresponding change in $d N\left(>\tau_{0}\right) / d z$ will not be large. For example, in the range of optical depths shown in Figure 3, setting $T_{S}=10^{2} \mathrm{~K}$ increases the number density of lines by factor of only $\sim 2$.

Figure 4 shows the variation of $d N\left(>\tau_{0}\right) / d z$ with IGM properties at $z=8$. The solid curves assume $J_{-21}=1$, the dotted curves assume $J_{-21}=0$, and the dashed curves assume $J_{-21}=10$. Within each set, the left line assumes our standard IGM temperature and the right line assumes that $T_{\mathrm{IGM}}$ is an order of magnitude below the value given by equation (6). The steep rise as $\tau_{0}$ approaches $\tau_{\text {IGM }}$ is caused by the divergence of the relevant cross-section for each halo in this limit. We see that the radiation background can be inferred from the absorbing continuum while $T_{\text {IGM }}$ can be estimated from the statistics of deep lines. At small $\tau_{0}$, the distribution is primarily determined by the radiation field $J_{-21}$. On the other hand, $d N\left(>\tau_{0}\right) / d z$ depends much more strongly on $T_{\mathrm{IGM}}$ than on $J_{-21}$ at large $\tau_{0}$. Such large optical depths require a line of sight passing close to the halo center, where the gas density is high enough for collisional coupling between $T_{S}$ and $T_{K}$ to be effective, even without a substantial radiation field. The temperature dependence arises because a cool IGM has a smaller $M_{\text {fil }}$ and thus a larger number of low-mass halos with small $T_{\text {vir }}$ (and correspondingly large $\left.\tau_{0}\right)$.

Carilli et al. (2002) examined lines of sight through a cosmological simulation and estimated that $d N\left(\tau_{0}>0.02, z=10\right) / d z \sim 50$ and $d N\left(\tau_{0}>0.02, z=8\right) / d z \sim 4$. For their simulation, the average radiation background evolves between $J_{-21} \sim 1-4$ over this redshift range. Figure 4 shows that their $z=8$ result lies in the range we would predict for this radiation field, but their $z=10$ point is $\sim 4$ times higher than our prediction in Figure 3. [If we choose $T_{\text {IGM }}$ to precisely match the mass-averaged temperature at $z=10$ in the simulation of Carilli et al. (2002), the discrepancy is reduced to a factor of $\sim 3$.] A difference in results should be expected given that the two calculations are complementary and do not consider the same population of absorbers. The baryonic mass per particle in the simulation was $10^{5.7} \mathrm{M}_{\odot}$ (Gnedin 2001), while even the largest of our minihalos have baryonic masses $\sim 10^{6.7} \mathrm{M}_{\odot}$. Our formalism therefore probes objects of much smaller mass than does the simulation; on the other hand, the simulation probes the filamentary structure of the IGM which is not adequately described by our formalism. Moreover, the simulation includes non-uniform heating and radiation. Overall, it appears that filaments and minihalos provide comparable contributions to the line statistics, with filaments being relatively more important at higher redshifts.

Another useful quantity is the number of intersections per redshift interval with observed equivalent widths greater than a fixed value, $d N(>E W) / d z$. In Figure 5 we show this for the same redshifts and IGM characteristics as in Figure 3. Again, disks only dominate the 
number counts for very large equivalent widths. In contrast to the optical depth statistics, we find little evolution of $d N(>E W) / d z$ with redshift. This is caused in part because our definition of the equivalent width removes the effect of $\tau_{\text {IGM }}$ (which decreases rapidly with cosmic time). In addition, the small halos that provide large $\tau_{0}$ at high- $z$ are narrow and therefore do not have substantial equivalent widths. As a result, the simultaneous increases in $M_{\text {fil }}$ and minihalo density with cosmic time appear to roughly balance each other. We do not show the dependence of the equivalent width statistics on $J_{-21}$ and $T_{\text {IGM }}$ because it exhibits the same trends as $d N\left(>\tau_{0}\right) / d z$, although at a weaker level.

\subsection{Synthetic Spectra}

Our model can also be used to generate Monte Carlo realizations of absorption spectra for illustrative purposes. The probability $P(z)$ that a line of sight intersects an object in a redshift interval $d z$ centered on $z$ is given by equation (15), with $A\left(M_{h}, z\right)$ simply detemined by the maximum impact parameter allowed in the calculation. (We find that choosing $\alpha_{\max }=5 r_{\text {vir }}$ is sufficient to include all observable absorption lines from minihalos.) To generate a spectrum, we choose redshift intervals $\Delta z$ small enough so that $P(z)<0.01$. For each bin, we generate a random number to determine if an intersection takes place. If one occurs, we choose $M_{h}$ randomly using the probability distribution $d n_{h} / d M_{h}$ and then randomly select the impact parameter with an equal probability per cross-sectional area of the halo. Note that this method does not include clustering of minihalos and in principle allows overlap between minihalos centered in nearby bins (although in practice such overlap is highly insignificant).

Sample transmission curves generated in this way and smoothed to $1 \mathrm{kHz}$ resolution are shown in Figure 6. Here the transmission $T$ is defined as $T=1-\exp \left(-\tau_{\nu}\right)$. In each panel, we show the portion of a spectrum from $z=8.25$ to $z=7.75$. Each panel is generated from the same set of intersected halos, but assumes a different radiation field in the calculation of the optical depths $\left(J_{-21}=0,1\right.$, and 10 , from top to bottom). The figure explicitly shows that by coupling $T_{S}$ and $T_{K}$, a radiation field can strongly decrease both the absorption of the diffuse IGM and of the minihalos. [For reference, $T_{\text {IGM }}(z=8)=3162 \mathrm{~K}$ in our model.] The strongest absorption is caused by a $2 \times 10^{6} \mathrm{M}_{\odot}$ minihalo at $z=8.2$, with $\alpha=0.35 r_{\text {vir }}$. Close inspection reveals that most absorption lines are surrounded by a small region with transmission greater than that of the diffuse IGM. Such features result from the steep velocity gradients in the infall region of a halo, which cause the optical depth to fall below $\tau_{\text {IGM }}$ by small factor (see Figure 1).

Similarly, one can generate realizations of spectra including disk intersections. Because 
disk intersections are rare (see Figure 3), we performed a Monte Carlo procedure on the interval between $z=8.25$ and $z=7.75$ sixteen times in order to find a line of sight passing through a disk with $\tau_{0}>0.1$. [Figure 3 shows that for disks, $d N\left(\tau_{0}>0.1\right) / d z \approx 0.09$ at $z=8$. On average, we would therefore expect one such intersection per 22 realizations of this redshift interval.] In fact, the resulting system has $\tau_{0}=3.76$. We then added this system to an independently generated minihalo field, and we show the resulting spectrum in Figure 8. In order to contrast the effects of disk and minihalo intersections, we switch to logarithmic axes and plot the flux decrement, $1-T=\exp \left(-\tau_{\nu}\right)$, instead of the transmission. The strong feature is caused by the disk, while the much weaker features to the right are caused by minihalos. Although such lines of sight are obviously rare, we see that disks can in general be clearly distinguished from the minihalo population because they have much larger peak optical depths and equivalent widths. Note that the line width here is a direct result of our assumption that $T_{K}=10^{3} \mathrm{~K}$. Most damped Ly $\alpha$ absorbers at lower redshifts show more than one velocity component of somewhat narrower intrinsic width (Briggs et al. 2001; Lane \& Briggs 2001).

In principle, another way to detect the above features is to integrate the total amount of absorption they cause over a large bandwidth. Unfortunately, the simulated line of sight shown in Figure 6 implies that minihalos add only a small correction $(\sim 5-10 \%$, depending

on the radiation field) to the already small absorption $\left(\tau_{\text {IGM }} \lesssim 0.01\right)$ caused by the diffuse IGM, and we predict that the identification of individual lines is a more powerful method of studying the minihalo population.

\section{Discussion}

We have shown that absorption at the $21 \mathrm{~cm}$ transition of neutral hydrogen in both minihalos and protogalactic disks at high-redshifts can cause non-negligible absorption in the low-frequency spectra of high-redshift radio sources. Using a semi-analytic approach, we have found that the density of minihalo absorption features is primarily a function of the kinetic temperature of the IGM (which determines the minimum mass of the minihalos) and the radiation background (which couples the spin and kinetic temperatures of the hydrogen gas). We predict that the average radiation background can be measured by the continuum absorption and the number density of very weak lines, while the IGM temperature can be measured by the density of narrow, relatively strong absorption lines. Optical depths reach $\tau_{0} \sim 0.02$ for objects common enough to appear along typical lines of sight. Studies of absorption radio spectra therefore offer a probe of the era before reionization and can help constrain the history of early star formation. We find, unsurprisingly, that disks are only 
rarely intersected but tend to have much higher optical depths.

The principal shortcomings of our semi-analytic approach are its inability to accurately describe both the filamentary structure of the IGM and the spatial clustering of halos. Using a simulation, Carilli et al. (2002) have also studied $21 \mathrm{~cm}$ absorption along lines of sight to high-redshift radio sources. While the mass resolution of their simulation was not sufficiently fine to include the minihalo population, they were able to capture the geometry and structure of filaments. They find that both the typical optical depths and the number densities of filament absorption lines are comparable to or slightly larger than our estimates for those from minihalos. An observed absorption spectrum will then be a mixture of lines from these two populations of absorbers. The clustering of minihalo lines may yield information on bias or feedback from nearby cooled objects, but a proper study of such an effect will require numerical simulations or more detailed semi-analytic models (e.g., Scannapieco \& Barkana 2002).

Can these signals be detected by future low-frequency radio telescopes? The transmission curves in $§ 3.2$ ignore noise. In a real observation, the noise level will be determined by a combination of the detector characteristics and the brightness of the background source. Because the specifications of the next generation of detectors are not yet settled, we have chosen not to present results specifically tailored to any particular instrument. Instead, we can simply estimate the required source brightness, $S_{\min }$, in order to observe a single absorption feature, given the detector's effective area $A_{\text {eff }}$, system temperature $T_{\text {sys }}$, and channel width $\Delta \nu_{\mathrm{ch}}$, for a specified signal-to-noise ratio $S / N$ and integration time $t$. In the small $\tau_{0}$ limit and under the assumption that $\Delta \nu_{\text {ch }} \ll \Delta \nu_{\text {obs }}$, the result is

$$
S_{\min }=12 \mathrm{mJy}\left(\frac{S / N}{5}\right)\left(\frac{0.02}{\tau_{0}}\right)\left(\frac{2 \times 10^{3} \mathrm{~m}^{2} \mathrm{~K}^{-1}}{A_{\mathrm{eff}} / T_{\mathrm{sys}}}\right)\left(\frac{10 \text { days }}{t}\right)^{1 / 2}\left(\frac{\mathrm{kHz}}{\Delta \nu_{\mathrm{ch}}}\right)^{1 / 2} .
$$

Current designs for the SKA call for $A_{\text {eff }} / T_{\text {sys }} \sim 2 \times 10^{3} \mathrm{~m}^{2} \mathrm{~K}^{-1}$, while the Low Frequency Array $^{2}$ (LOFAR) is expected to have $A_{\text {eff }} / T_{\text {sys }} \sim 4.5 \times 10^{2} \mathrm{~m}^{2} \mathrm{~K}^{-1}$. A channel width of $1 \mathrm{kHz}$ should be achievable with LOFAR while maintaining a total spectral coverage $\sim 4 \mathrm{MHz}$ and with SKA while maintaining a total spectral coverage of $\sim 10 \mathrm{MHz}$.

Do objects of this brightness exist at sufficiently high redshifts? While we currently have no observational constraints, Carilli et al. (2002) have presented a plausibility argument for the existence of luminous radio-loud quasars at $z \gtrsim 7$. By extrapolating the radio galaxy luminosity function to high redshifts using the observed decline of optical quasars, they estimate $\gtrsim 2000$ sources on the sky of sufficient brightness to be useful. Furthermore, they

\footnotetext{
${ }^{2}$ See http://www.lofar.org/index.html.
} 
point out that a bright radio galaxy at $z=5.2$ has already been detected (van Breugel et al. 1999), and there is no obvious reason for a high- $z$ cutoff.

Because of their high optical depths, disks could be detected along lines of sight to much fainter sources. One particularly interesting type of source is a gamma-ray burst (GRB). While not sufficiently bright for study of minihalo absorption features (the typical flux is $\lesssim 1$ mJy; Ciardi \& Loeb 2000), GRBs can nevertheless be used to study intervening disks in which $\tau_{0} \gtrsim 1$. The smooth power-law spectrum of GRBs is an ideal continuum for these observations. In this case, the rarity of disks is not a limit because the host galaxy of each GRB is necessarily present at the redshift of the burst. If the GRB is embedded in a star forming disk then the corresponding (deep) $21 \mathrm{~cm}$ line can confirm the redshift of the GRB and provide information on the neutral hydrogen content of the host galaxy.

We thank B. Gaensler for helpful discussions. This work was supported in part by NASA grants NAG 5-7039, 5-7768, and NSF grants AST-9900877, AST-0071019 for AL. SRF acknowledges the support of an NSF graduate fellowship.

\section{REFERENCES}

Allison, A. C. \& Dalgarno, A. 1969, ApJ, 158, 423

Barkana, R. \& Loeb, A. 2001, Phys. Rep., 349, 125

Barkana, R. \& Loeb, A. 2002, ApJ, submitted (astro-ph/0204139)

Becker, R. H. et al. 2001, AJ, 122, 2850

Bertschinger, E. 1985, ApJS, 58, 39

Briggs, F. H., de Bruyn, A. G., \& Vermeulen, R. C. 2001, A\&A, 373, 113

Carilli, C. L., Lane, W., de Bruyn, A. G., Braun, R., \& Miley, G. K. 1996, AJ, 111, 1830

Carilli, C. L., Gnedin, N. Y., \& Owen, F. 2002, ApJ, in press (astro-ph/0205169)

Ciardi, B., Ferrara, A., \& Abel, T. 2000, ApJ, 533, 594

Ciardi, B. \& Loeb, A. 2000, ApJ, 540, 687

Dickey, J. M. \& Lockman, F. J. 1990, ARA\&A, 28, 215

Di Matteo, T., Perna, R., Abel, T., \& Rees, M. J. 2002, ApJ, 564, 576 
Fan, X., Narayanan, V. K., Strauss, M. A., White, R. L., Becker, R. H., Pentericci, L., \& Rix, H. 2002, AJ, 123, 1247

Field, G. B. 1958, Proc. IRE, 46, 240

Field, G. B. 1959a, ApJ, 129, 525

Field, G. B. 1959b, ApJ, 129, 551

Gnedin, N. Y. 2000, ApJ, 535, 530

Gnedin, N. Y. 2001, MNRAS, submitted (astro-ph/0110290)

Gnedin, N. Y. \& Hui, L. 1998, MNRAS, 296, 44

Gunn, J. E. \& Peterson, B. A. 1965, ApJ, 142, 1633

Haiman, Z., Rees, M. J., \& Loeb, A. 1997, ApJ, 476, 458

Haiman, Z., Abel, T., \& Rees, M. J. 2000, ApJ, 534, 11

Iliev, I. T., Shapiro, P. R., Ferrara, A., \& Martel, H. 2002, ApJ, submitted (astro$\mathrm{ph} / 0202410)$

Jenkins, A., Frenk, C. S., White, S. D. M., Colberg, J. M., Cole, S., Evrard, A. E., Couchman, H. M. P., \& Yoshida, N. 2001, MNRAS, 321, 372

Kumar, A., Padmanabhan, T., \& Subramanian, K. 1995, MNRAS, 272, 544

Lane, W. M. \& Briggs, F. H. 2001, ApJ, 561, L27

Loeb, A. 1996, ApJ, 459, L5

Madau, P., Meiksin, A., \& Rees, M. J. 1997, ApJ, 475, 429

Mo, H. J., Mao, S., \& White, S. D. M. 1998, MNRAS, 295, 319

Navarro, J. F., Frenk, C. S., \& White, S. D. M. 1997, ApJ, 490, 493

Press, W. H. \& Schechter, P. 1974, ApJ, 187, 425

Ricotti, M., Gnedin, N. Y., \& Shull, J. M. 2002, ApJ, in press; astro-ph/0110432

Scannapieco, E. \& Barkana, R. 2002, ApJ, 571, 585

Scott, D. \& Rees, M. J. 1990, MNRAS, 247, 510 
Shaver, P. A., Windhorst, R. A., Madau, P., \& de Bruyn, A. G. 1999, A\&A, 345, 380

Tozzi, P., Madau, P., Meiksin, A., \& Rees, M. J. 2000, ApJ, 528, 597

van Breugel, W., De Breuck, C., Stanford, S. A., Stern, D., Röttgering, H., \& Miley, G. 1999, ApJ, 518, L61

Wouthuysen, S. A. 1952, AJ, 57, 31 


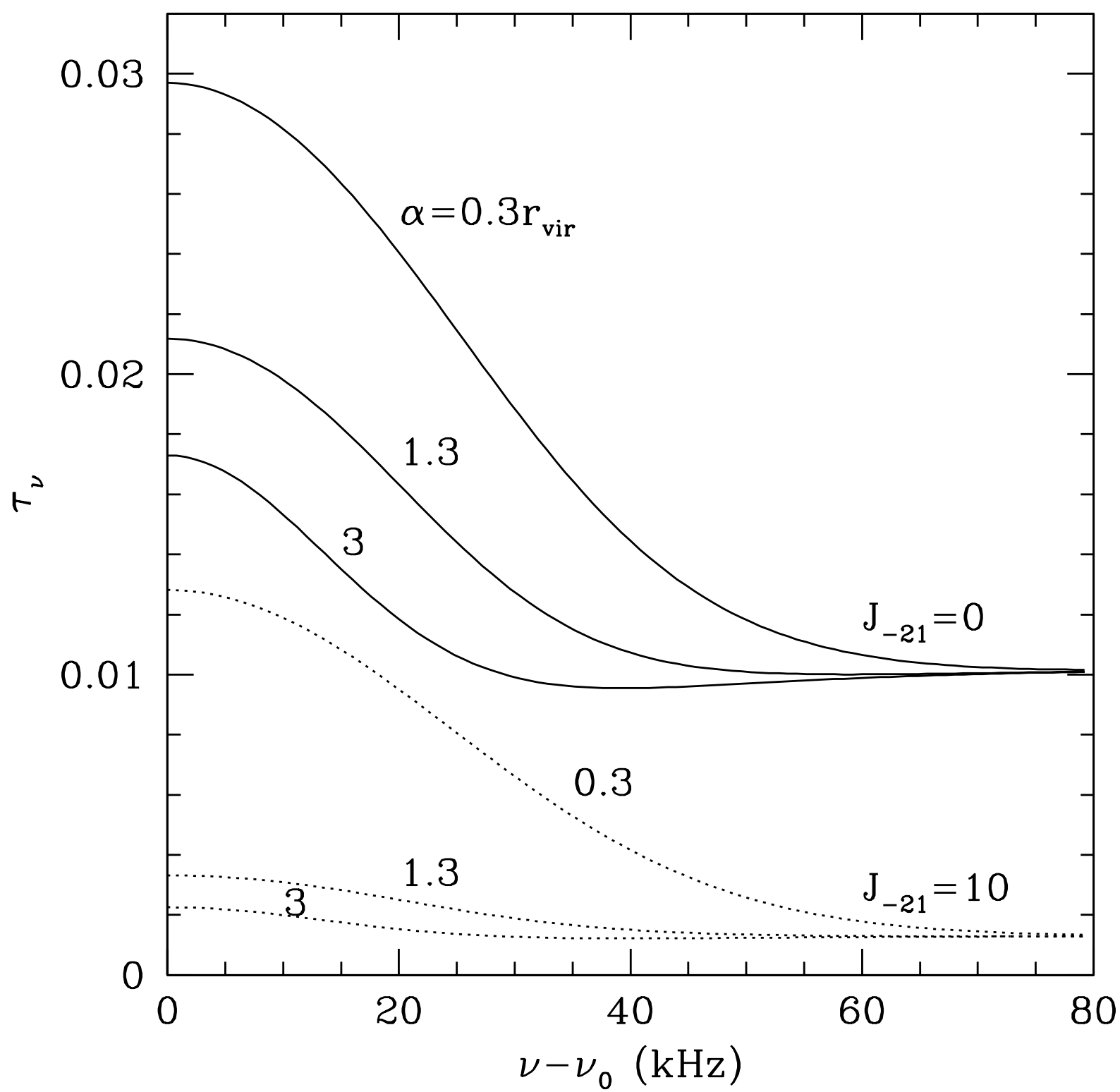

Fig. 1.- Optical depth profiles for a $M_{h}=5 \times 10^{6} \mathrm{M}_{\odot}$ minihalo at $z_{h}=10$ embedded in a heated IGM. Each set of profiles assumes $\alpha=0.3,1.3$, and $3 r_{\text {vir }}$, from top to bottom. The solid curves assume a heated IGM and $J_{-21}=0$, while the dotted lines show results for $J_{-21}=10$. 


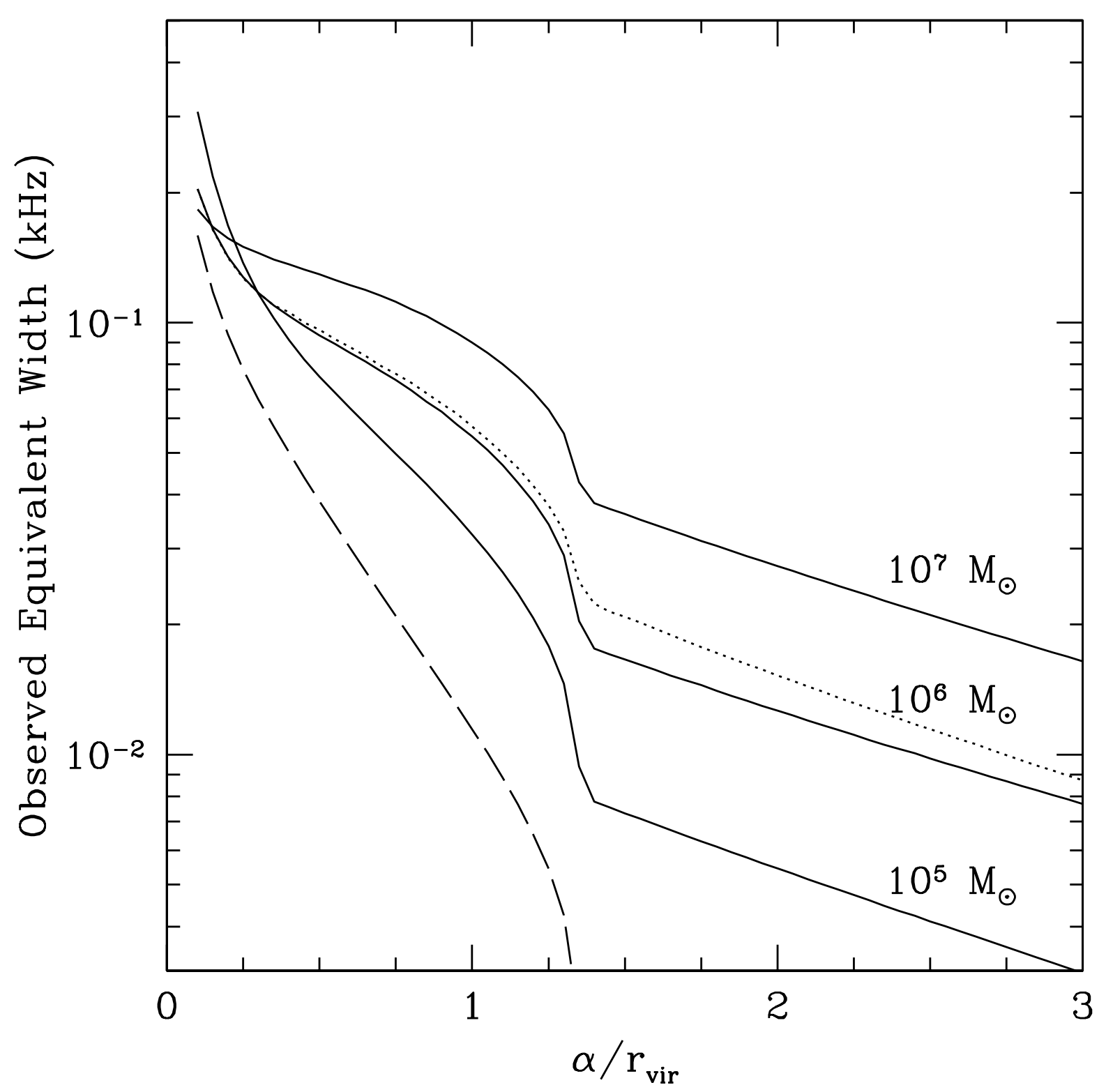

Fig. 2.- Observed equivalent width as a function of impact parameter $\alpha$. Solid curves show results for $M_{h}=10^{7}, 10^{6}$, and $10^{5} \mathrm{M}_{\odot}$ from top to bottom, with $J_{-21}=0$ and a heated IGM. The dotted curve shows results for $M_{h}=10^{6} \mathrm{M}_{\odot}$ and no IGM heating, while the dashed curve shows results for the same mass, IGM heating, and a radiation background $J_{-21}=10$. All curves assume $z_{h}=8$. 


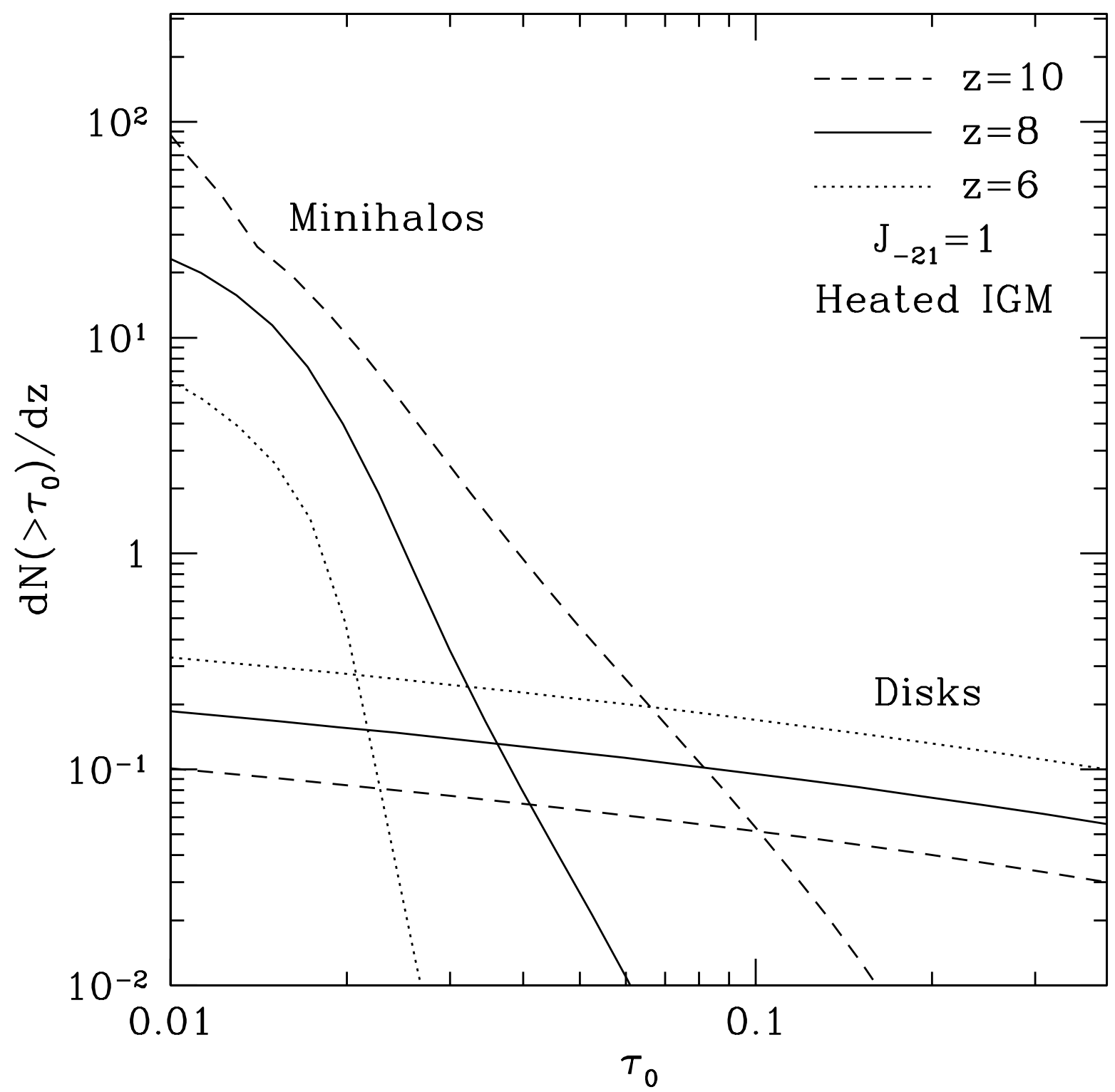

Fig. 3.- Number of systems with observed central optical depth greater than $\tau_{0}$ intersected per redshift interval, as a function of $\tau_{0}$. The dashed, solid, and dotted curves show results for $z=10,8$, and 6 , respectively. The two sets of lines describe minihalos and protogalactic disks. Minihalo calculations assume $J_{-21}=1$ and a heated IGM. 


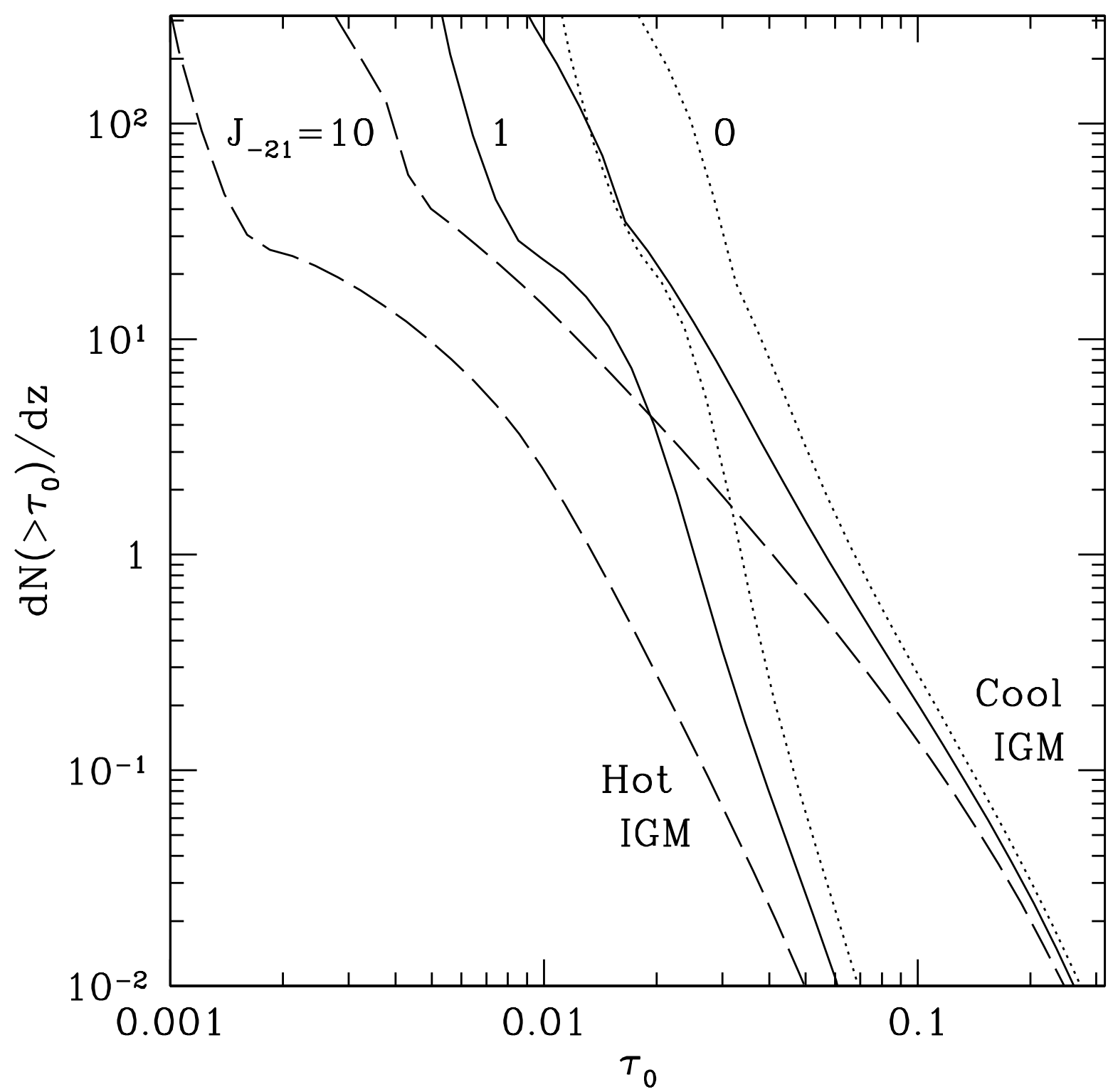

Fig. 4.- Number of systems with observed central optical depth greater than $\tau_{0}$ intersected per redshift interval at $z=8$. The solid curves assume $J_{-21}=1$, the dotted curves assume $J_{-21}=0$, and the dashed curves assume $J_{-21}=10$. Within each set, the leftmost curve assumes our standard IGM temperature and the rightmost curve assumes $T_{\mathrm{IGM}}$ is an order of magnitude below that of equation (6). 


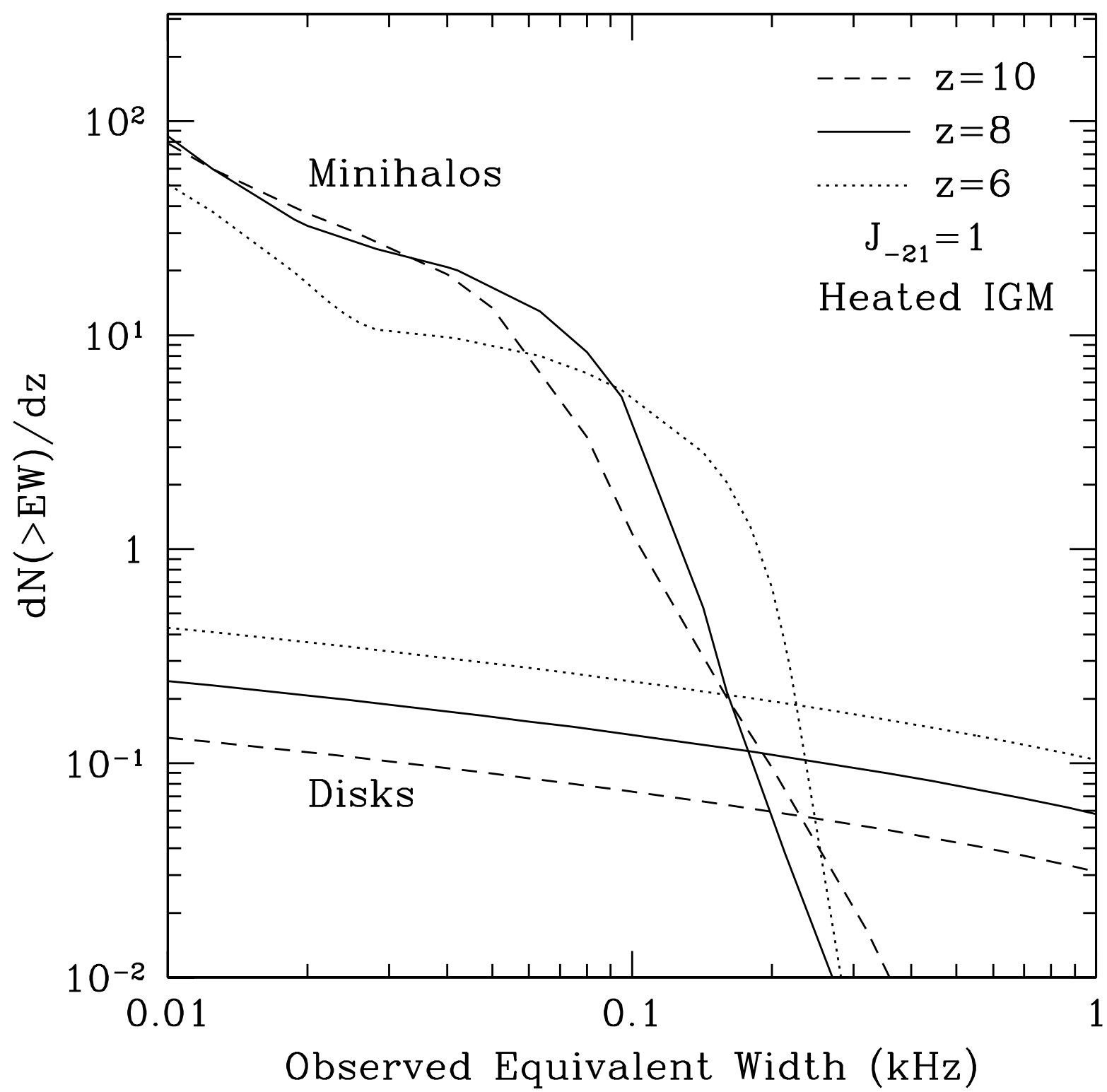

Fig. 5.- Number of systems with observed equivalent width greater than a threshold intersected per redshift interval, as a function of the threshold value. The dashed, solid, and dotted curves show results for $z=10,8$, and 6 , respectively. The two sets of lines describe minihalos and protogalactic disks. All minihalo calculations assume a heated IGM and $J_{-21}=1$. 


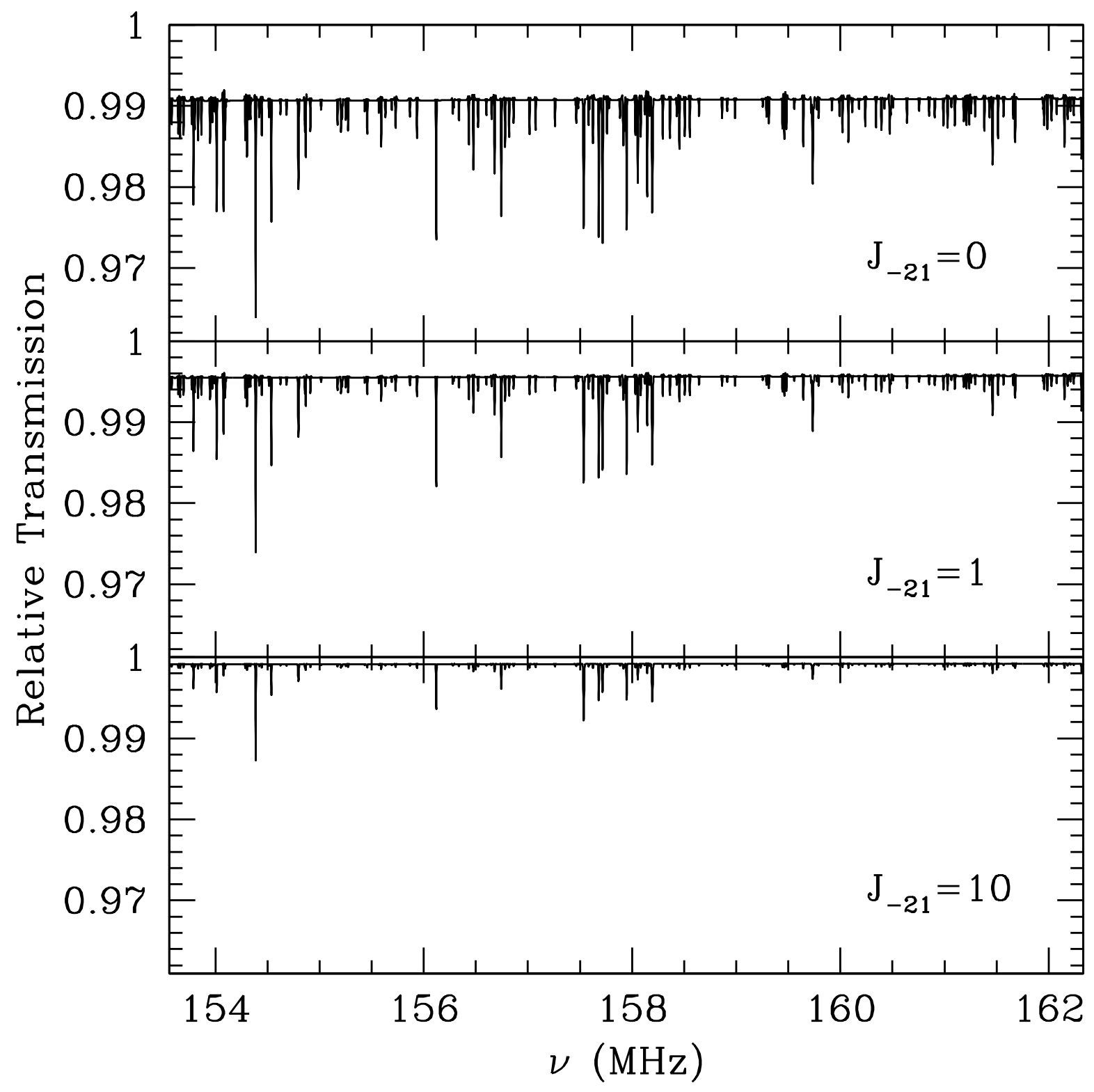

Fig. 6.- Simulated transmission along a line of sight to a distant radio source assuming absorption by intervening minihalos. All panels are generated using the same realization of the minihalo mass field and assume a heated IGM. The panels show $J_{-21}=0,1$, and 10 , from top to bottom. 


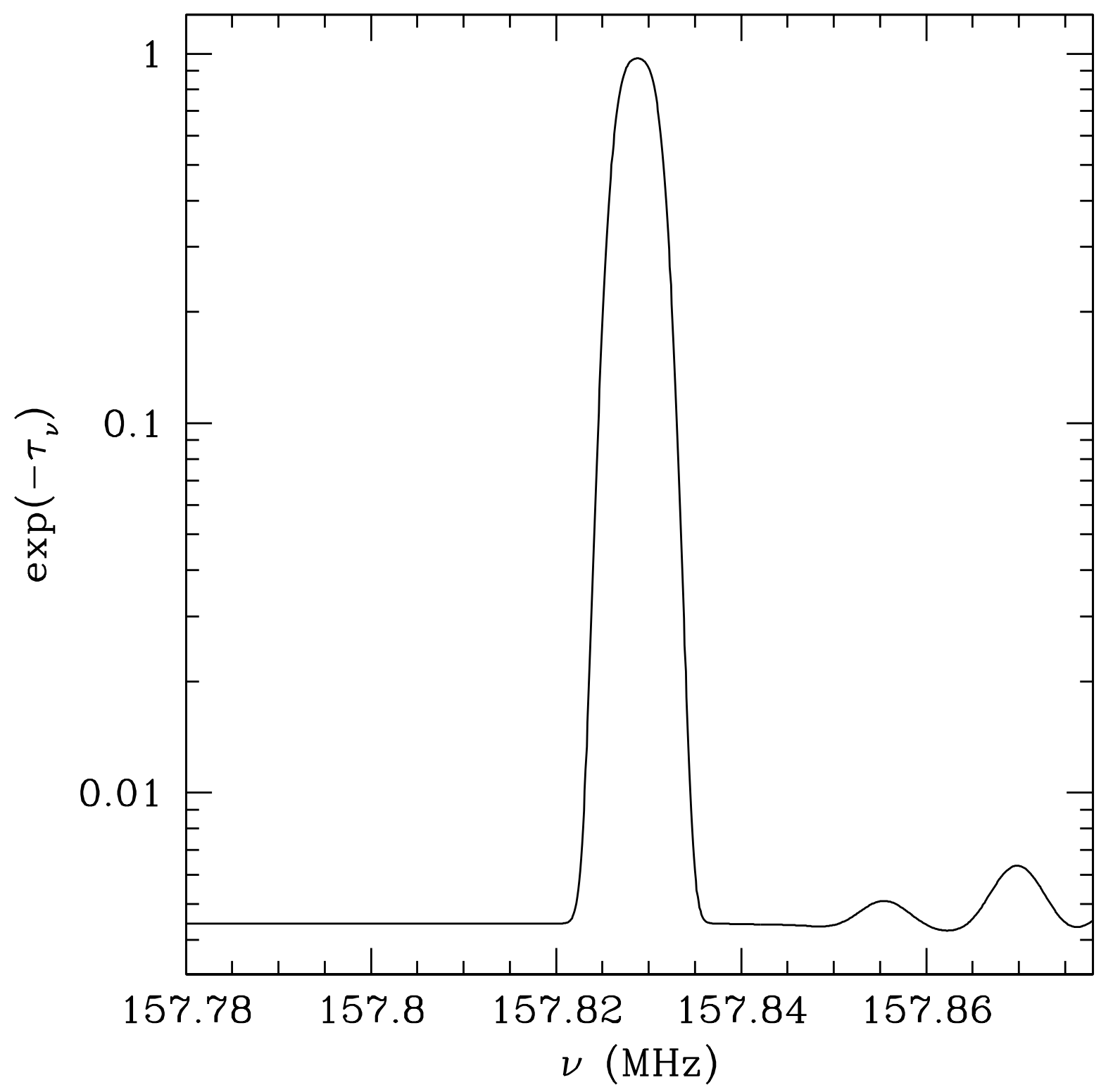

Fig. 7.- Simulated flux decrement profile along a sightline to a distant radio source chosen to intersect a protogalactic disk. The accompanying minihalo mass field assumes a heated IGM and $J_{-21}=1$. 